\title{
Probability Density Function of Liver disease with or without Cirrhosis in Time Domains
}

\author{
Sunpreet Kaur \\ PG student Dept. electronics and communication \\ engineering, Chandigarh University \\ Gharuan, Punjab, India 140413
}

\author{
Vaibhav Arora \\ Assistant Professor Dept. electronics and \\ communication engineering, Chandigarh \\ University \\ Gharuan, Punjab, India 140413
}

\begin{abstract}
This paper attempts to estimate the pdf probability density function of various parameters of liver disease with cirrhosis or without cirrhosis. Some parameters are analysed and out of those best four parameters are taken into consideration. Parameters include ratio of ALT and AST (alanine aminotransferases and aspartate aminotransferases), ALB (serum Albumin), Bil (bilirubin), Prot (total protein) Patients belonging to different age groups are analysed. The pdf has been carried out in time domains. Various distributions have been tested to get a good fit for most of the data. It is observed that there is different distribution for different parameters.
\end{abstract}

\section{General Terms}

This method is used for classification purpose. This is the another method of classification of data set in probability density function. It is used as a clinical data to design liver disease cirrhosis detection system.

\section{Keywords}

Liver Disease; Cirrhosis; probability distribution function; AST (aspartate amino transferases); ALT (alanine amino transferases); ALB (serum Albumin); Bil (bilirubin).

\section{INTRODUCTION}

Liver is a vital organ of human's body. It is located in upper right quadrant of Abdomen. The liver has wide range of functions generally cite it being around 500. Liver plays an important role in human body like decomposition of red blood cells, protein production, blood clotting to cholesterol, produce bile which helps indigestion. Many bio chemical reactions occur in liver like synthesis and breakdown of small and complex molecules. A variety of illness can affect the function of liver. One of the disease is Cirrhosis which occurs when normal liver replaced by scar tissues as a result of chronic liver disease. Cirrhosis is caused by alcoholism, hepatitis $\mathrm{b}$, hepatitis $\mathrm{c}$, and fatty liver. Cirrhosis is irreversible. Treatment usually focuses on preventing progress. Liver transplantation is the only option of advance stage of cirrhosis.

In this paper we have attempted to estimate the Probability Density Function (pdf) of different parameters. In this we have Probability Density Function for parameters of liver disease. Probability density function (PDF), or density of a continuous random variable, is a function that describes the relative likelihood for this random variable to take on a given value. Probability distribution is defined as a function over general sets of values. The probability density function is nonnegative everywhere, and its integral over the entire space is equal to one.

Hence, if $\mathrm{Fx}$ is the cumulative distribution function of $\mathrm{X}$, then:

$\mathrm{FX}(\mathrm{X})=\int_{-\infty}^{\mathrm{X}} \mathrm{fX}(\mathrm{u}) d \mathrm{u}$

And (if $\mathrm{fX}$ is continuous at $\mathrm{x}$ )

The probability density function is:

$\mathrm{fX}(\mathrm{x})=\frac{d}{d \mathrm{u}} \mathrm{FX}(\mathrm{x})$

This paper is organized into the following five sections. The first section describes parameters of liver disease with no cirrhosis used in this study. In the same section results from the time domain estimation has been shown. The second section describes parameters of liver disease with cirrhosis used in this study. In the same section results from the time domain estimation has been shown. Then conclusions are summarized then finally acknowledgement is mentioned.

\section{ESTIMATION OF PARAMETERS RESULTS IN LIVER DISEASE WITH NO CIRRHOSIS IN THE TIME DOMAIN}

\subsection{Data Description}

The present study has been done on patients having liver disease with cirrhosis and without cirrhosis. Data set include one hundred three patients. Data is taken from Department of Gastroenterology and Department of Hepatology, Nehru Hospital, PGIMER, Chandigarh. Patients are of different ages. In this routine blood tests including serum alanine and aspartate aminotransferases (ALT and AST), alkaline phosphatase (ALP), direct and total serum bilirubin, total protein and albumin, prothrombin time and other hematological parameters including hemoglobin, platelets, white blood cell counts, red blood cell indices and peripheral blood film were obtained from PGIMER laboratories. On the basis of clinical and biochemical evidence 80 out of 103 alcoholics $(77.6 \%)$ were found to have liver disease. Out of 80 alcoholic patients $16(20 \%)$ had fatty liver disease, 5 (6.2\%) had hepatitis and $58(72.5 \%)$ had liver cirrhosis. 46 $(57.5 \%)$ patients with liver cirrhosis had decompensate cirrhosis and $12(15 \%)$ patients had acute chronic liver failure (ACLF). 67 (74.4\%) alcoholics had liver disease and 47 (52.2 $\%)$ of them had liver cirrhosis.

Out of 103 patients 68 (66\%) alcoholics had education level of high school or below. Binge drinking was observed in 71 $(68.9 \%)$ cases. $37(35.9 \%)$ alcoholics had history of a drink 
first thing in the morning to steady nerves or get rid of a hangover (eye-opener).

Regarding the type of liquor most commonly consumed, about $55(53.4 \%)$ alcoholics consumed only foreign liquor, $34(33 \%)$ consumed locally-made liquor, and 14 (13.6\%) consumed both the types of liquor. The most common type of alcoholic beverage for drinkers in this study was whisky, followed by locally-made liquor

Dataset include various parameters and PDF estimation of following parameters is calculated:

(i)Ratio of ALT and AST (alanine and aspartate aminotransferases) (ii) Bil (bilirubin) (iii) ALB (serum Albumin) (iv) Prot (total protein) (v) ALP (Alkaline Phosphatase) (vi) PTI (Prothrobin index) (vii) TLC

\subsection{Goodness of Fit Test}

Data is divided into three categories as liver disease with cirrhosis, liver disease without cirrhosis, and patients having no liver disease. In the time domain, Chi-squared, Anderson Darling, and Kolmogorov-Smirnov tests are applied on the dataset containing patients having liver disease with or without cirrhosis

The histogram plots of liver disease with no Cirrhosis have been depicted in Figs. 2(a)-2(d).
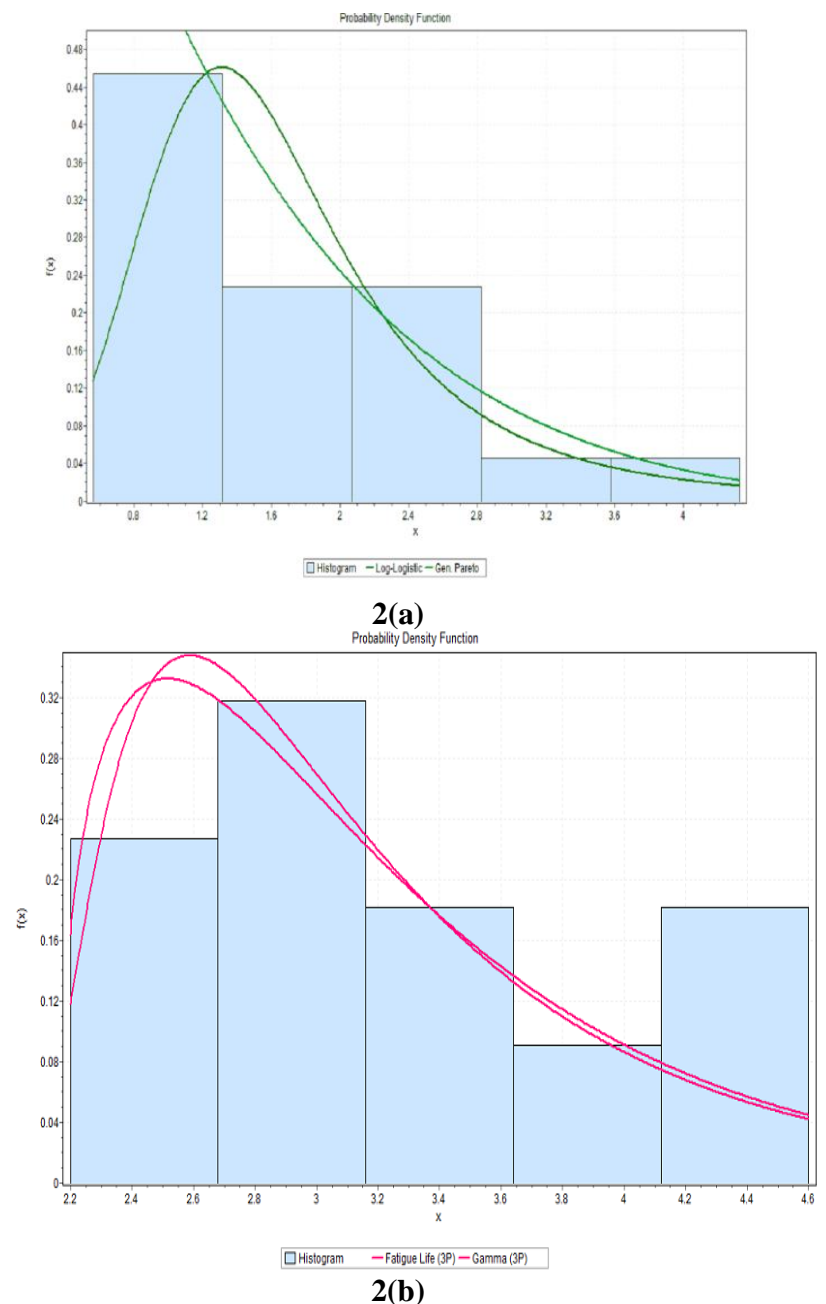

2(b)
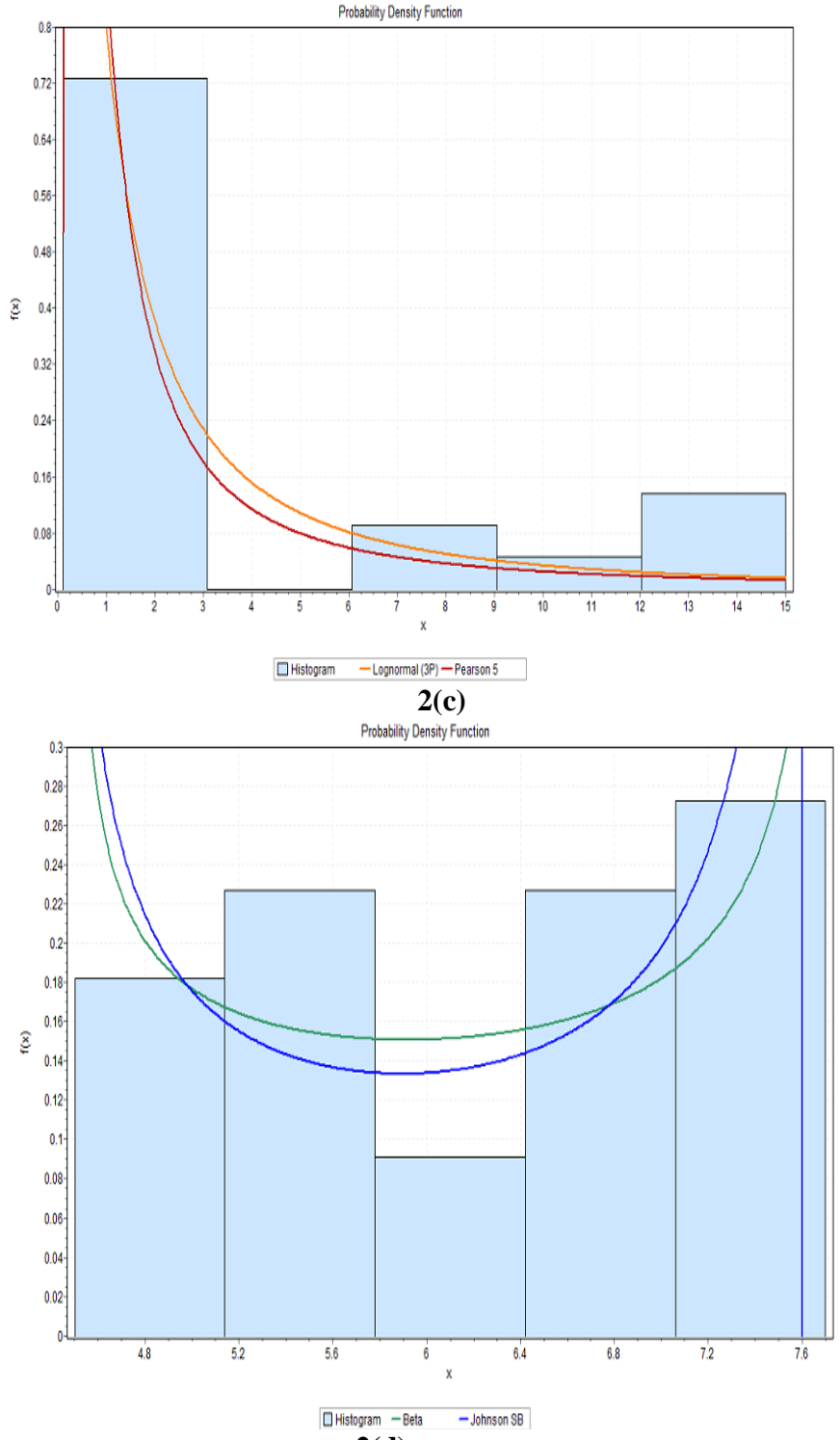

2(d)

Fig.2.Histogram Plots and Best Fitting Curves in Time Domain for the liver disease with no cirrhosis having following parameters are shown (a) Ratio of ALT and AST (alanine and aspartate aminotransferases) (b) ALB (serum Albumin) (c) Bil (bilirubin) (d) Prot (total protein).

It is observed that every parameter necessary for liver disease have different distribution. For ratio of AST and ALT parameter, Log Logistic and Gen. Pareto are best distributions. Formula for these distributions is as follows:

The formula for Log Logistic:

$\alpha$ - continuous shape parameter $\quad(\alpha>0)$

$\beta$ - continuous scale parameter $(\beta>0)$

$\mathrm{f}(\mathrm{x} ; \alpha, \beta)=\frac{(\beta / \alpha)(\mathrm{x} / \alpha)^{\wedge} \beta-1}{\left(1+(\mathrm{x} / \alpha)^{\wedge} \beta\right)^{\wedge} 2}$

Whose expectation is

$\mathrm{E}(\mathrm{X})=\alpha \mathrm{b} / \sin \mathrm{b}, \quad \quad \beta>1$, 
The pdf for Gen. Pareto:

$f(x)=\left\{\frac{1}{\sigma}\left(1+\kappa \frac{(x-\mu)}{\sigma}\right)^{\wedge}-1-1 / \kappa \quad \kappa \neq 0\right.$

$f(x)=\left\{\frac{1}{\sigma} \exp \left(-\frac{x-\mu}{\sigma}\right) \quad \kappa=0\right.$

$\kappa$ - Continuous shape parameter

$\mu$ - Continuous scale parameter $(\sigma>0$ )

$\sigma$ - Continuous location parameter

Pdf for Fatigue Life (3P):

$F(x)=\frac{\sqrt{(x-\gamma) / \beta+\sqrt{ } \beta /(x-\gamma)}}{2 \alpha(x-\gamma)} \cdot \varphi\left(\frac{1}{\alpha}\left(\sqrt{\frac{x-\gamma}{\beta}}-\left(\sqrt{ } \frac{\beta}{(x-\gamma}\right)\right)\right.$

Pdf for Gamma 3P is:

$f(x)=\frac{(x-\gamma)^{\wedge \alpha}-1}{\beta^{\wedge} \alpha \Gamma(\alpha)} \exp (-(x-\gamma) / \beta)$

Pdf for Beta:

$\alpha$ 1- continuous shape parameter $\left(\begin{array}{ll}\alpha & 1>0\end{array}\right)$ $\alpha 2$ - continuous shape parameter $(\alpha 2>0)(a, b)$-continuous boundary parameters $(\mathrm{a}<\mathrm{b})$

Domain

$\mathrm{a} \leq \mathrm{x} \leq \mathrm{b}$

$\mathrm{f}(\mathrm{x})=\frac{1}{\mathrm{~B}(\alpha 1, \alpha 2)} \frac{(\mathrm{x}-\mathrm{a})^{\wedge} \alpha 1-1(\mathrm{~b}-\mathrm{x})^{\wedge \alpha 2}-1}{(\mathrm{~b}-\mathrm{a})^{\alpha 1}+\alpha 2-1}$

$\gamma<\mathrm{X}<+\infty$

Pdf for Lognormal:

Domain

$\gamma<\mathrm{x}<+\infty$

$\mathrm{f}(\mathrm{x})=\frac{\exp \left(-\frac{1}{2}\left(\frac{\ln (\mathrm{x}-\gamma)-\mu}{\sigma}\right)^{2}\right)}{(\mathrm{x}-\gamma) \sigma \sqrt{2 \pi}}$

Table 1. Goodness of fit test results in time domain

\begin{tabular}{|c|c|c|c|c|c|c|}
\hline $\begin{array}{l}\text { Sr } \\
\text { no. }\end{array}$ & Distribution & \multicolumn{2}{|c|}{$\begin{array}{l}\text { Kolmogoroov } \\
\text { Smirnov } \\
\text { (Test statistic) }\end{array}$} & \multicolumn{2}{|c|}{$\begin{array}{l}\text { Anderson } \\
\text { Darling } \\
\text { (Test } \\
\text { statistic) } \\
\end{array}$} & $\begin{array}{l}\text { Chi- } \\
\text { Squared } \\
\text { (Test } \\
\text { statistic }\end{array}$ \\
\hline \multicolumn{7}{|c|}{ AST/ ALT } \\
\hline 1 & $\begin{array}{l}\text { Log- } \\
\text { Logistic }\end{array}$ & \multicolumn{2}{|l|}{0.1462} & \multicolumn{2}{|c|}{0.53417} & 1.1006 \\
\hline 2 & Gen. Pareto & \multicolumn{2}{|l|}{0.14737} & \multicolumn{2}{|l|}{4.222} & N/A \\
\hline \multicolumn{7}{|c|}{ ALB } \\
\hline 1 & $\begin{array}{l}\text { Gamma } \\
(3 \mathrm{P})\end{array}$ & 0.09734 & \multicolumn{2}{|c|}{0.38178} & \multicolumn{2}{|c|}{0.30121} \\
\hline 2 & $\begin{array}{l}\text { Fatigue } \\
\text { Life(3P) }\end{array}$ & 0.10283 & \multicolumn{2}{|c|}{0.40146} & \multicolumn{2}{|c|}{0.25064} \\
\hline \multicolumn{7}{|l|}{ Bil } \\
\hline 1 & Pearson & 0.14571 & \multicolumn{2}{|c|}{0.60796} & \multicolumn{2}{|c|}{0.96156} \\
\hline 2 & $\begin{array}{l}\text { Lognor } \\
\text { mal }\end{array}$ & 0.15009 & \multicolumn{2}{|c|}{0.78377} & \multicolumn{2}{|c|}{2.1222} \\
\hline
\end{tabular}

\begin{tabular}{|l|l|l|l|l|}
\hline 1 & $\begin{array}{l}\text { Johnson } \\
\text { SB }\end{array}$ & 0.0878 & 7.3697 & N/A \\
\hline 2 & Beta & $\begin{array}{l}0.1000 \\
4\end{array}$ & 1.1733 & 0.00796 \\
\hline
\end{tabular}

\section{ESTIMATION OF PARAMETERS RESULTS IN LIVER DISEASE WITH CIRRHOSIS IN THE TIME DOMAIN}

On the basis of clinical and biochemical evidence 80 out of 103 alcoholics $(77.6 \%)$ were found to have liver disease. Out of 80 alcoholic patients $16(20 \%)$ had fatty liver disease, $5(6.2 \%)$ had hepatitis and $58(72.5 \%)$ had liver cirrhosis. $46(57.5 \%)$ patients with liver cirrhosis had decompensate cirrhosis and 12 (15\%) patients had acute chronic liver failure (ACLF). 67 $(74.4 \%)$ alcoholics had liver disease and $47(52.2 \%)$ of them had liver cirrhosis. The histogram plots of liver disease with no Cirrhosis have been depicted in Figs. 3(a)-3(d).
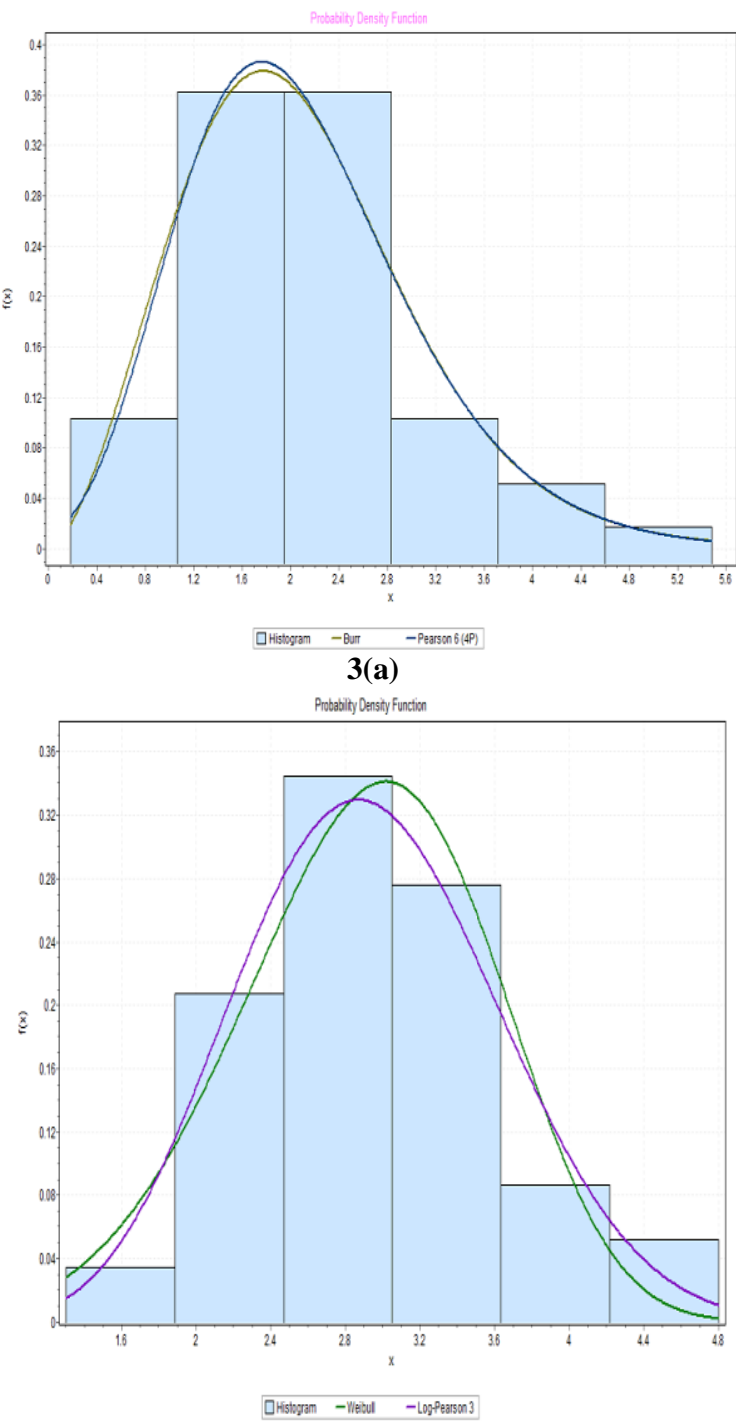

3(b) 

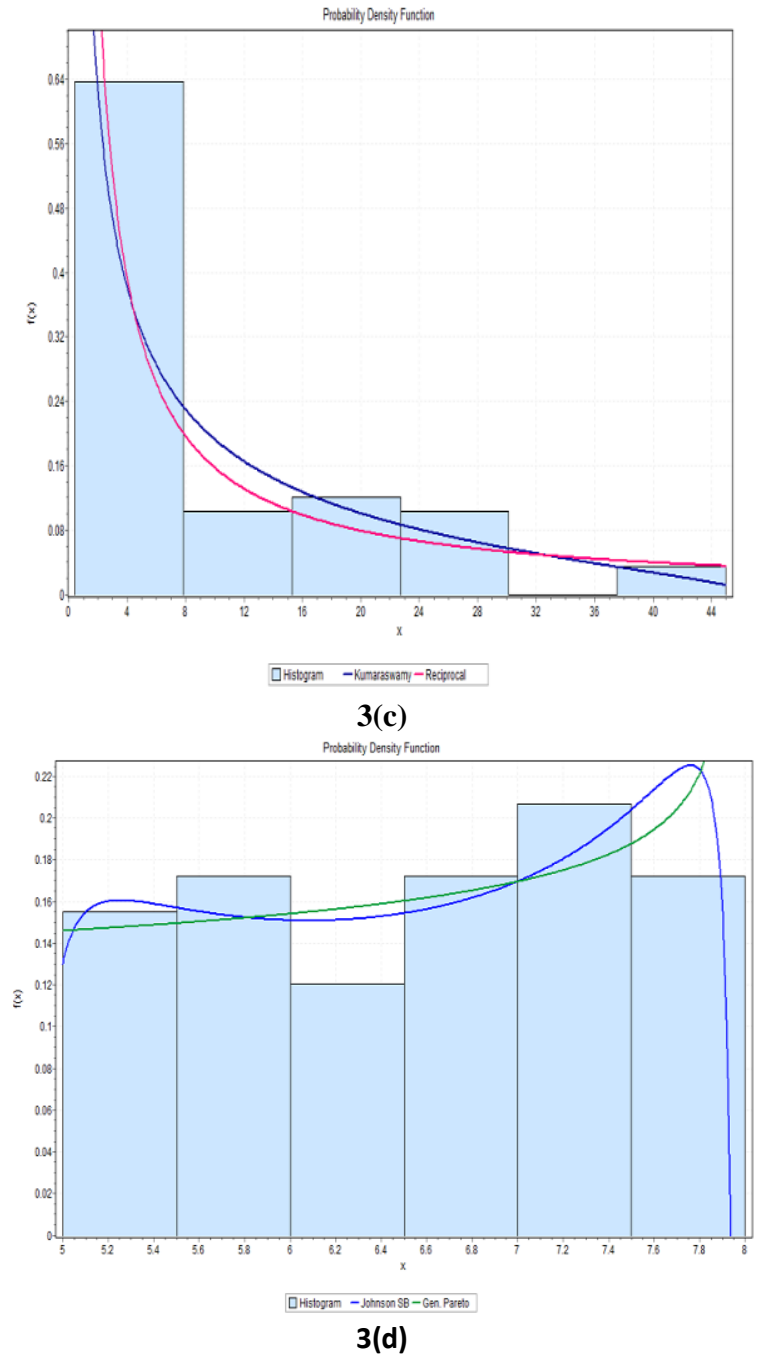

Fig 3 Histogram Plots and Best Fitting Curves in Time Domain for liver disease with cirrhosis having following parameters are shown (a) Ratio of ALT and AST (alanine and aspartate aminotransferases) (b) ALB (serum Albumin) (c) Bil (bilirubin) (d) Prot (total protein).

It is observed that every parameter necessary for liver disease have different distribution. For ratio of AST and ALT parameter, Pearson $6(4 \mathrm{P})$ and Burr are best distributions.

The pdf for Pearson 6 (4P) is:

$\mathrm{f}(x)=\frac{((\mathrm{x}-\gamma) / \beta)^{\wedge} \alpha 1-1}{\beta \mathrm{B}(\alpha 1, \alpha 2)(1+(\mathrm{x}-\gamma) / \beta)^{\wedge} \alpha 1+\alpha 2}$

Pdf for Burr is:

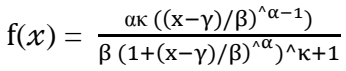

$\kappa$ - continuous shape parameter $(\kappa>0)$

$\alpha-\quad$ continuous shape parameter $(\alpha>0)$

$\beta$ - continuous scale parameter $(\beta>0)$

$\gamma$ - continuous location parameter $(\gamma=0$ continuous location parameter)

Pdf for Weibull:

$\left.\mathrm{f}(\mathrm{x})=\frac{\alpha}{\beta}\left(\frac{\mathrm{x}-\gamma}{\beta}\right)^{\wedge} \alpha-1 \exp (-(\mathrm{x}-\gamma) / \beta)^{\wedge} \alpha\right)$
Pdf for Kumaraswamy:

$f(x)=\frac{\alpha 1 \alpha 2 Z^{\alpha 1-1}\left(1-Z^{\alpha 1}\right)^{\alpha 2-1}}{(b-a)}$

Domain:

$$
\mathrm{a} \leq \mathrm{x} \leq \mathrm{b}
$$

Pdf for Reciprocal:

a,b- continuous boundary parameters $(0<\mathrm{a}<\mathrm{b})$

$f(x)=\frac{1}{x(\ln (b)-\ln (a))}$

The results are shown in TABLE II in which the test statistic of liver disease with cirrhosis is shown against a particular parameter and a particular test.

Fig.3. Histogram Plots and Best Fitting Curves for Time Domain Samples of LATERAL movements (a) female 1 (b) female 2(c) male 1 (d) male 2

Both the distributions fitted the data slightly better than each another. The results are shown in TABLE I in which the test statistic is shown against a particular gender and a particular test. However, a better fitting Burr (4P) distribution in sometimes can be a good alternative for hand movements EMG signals.

Table 2. Goodness of fit test results in time domain

\begin{tabular}{|c|c|c|c|c|c|}
\hline $\begin{array}{l}\text { Sr } \\
\text { no }\end{array}$ & $\begin{array}{l}\text { Distributi } \\
\text { on }\end{array}$ & $\begin{array}{l}\text { Kolmogo } \\
\text { Smirnov } \\
\text { statistic) }\end{array}$ & $\begin{array}{l}\text { ov } \\
\text { Test }\end{array}$ & \begin{tabular}{|l} 
Anderson \\
Darling \\
(Test \\
statistic)
\end{tabular} & $\begin{array}{l}\text { Chi- } \\
\text { Squared( } \\
\text { Test } \\
\text { statistic }\end{array}$ \\
\hline \multicolumn{6}{|c|}{ AST/ ALT } \\
\hline 1 & $\begin{array}{l}\text { Pearson } \\
6(4 \mathrm{P})\end{array}$ & 0.0567 & & 0.21938 & 6.4088 \\
\hline 2 & Burr & 0.05801 & & \begin{tabular}{|l|}
0.24359 \\
\end{tabular} & 6.3109 \\
\hline \multicolumn{6}{|c|}{$\overline{\mathrm{ALB}}$} \\
\hline \multicolumn{2}{|c|}{\begin{tabular}{l|l}
1 & Weibull
\end{tabular}} & 0.06791 & \multicolumn{2}{|c|}{0.48758} & 4.0503 \\
\hline 2 & Log-Pearson 3 & 0.07009 & \multicolumn{2}{|c|}{0.31619} & 3.168 \\
\hline \multicolumn{6}{|c|}{ Bil } \\
\hline 1 & $\begin{array}{l}\text { Kumarasw } \\
\text { amy }\end{array}$ & 0.07521 & \multicolumn{2}{|c|}{8.0632} & N/A \\
\hline 2 & Reciprocal & 0.07559 & \multicolumn{2}{|c|}{\begin{tabular}{|l|}
1.2973 \\
\end{tabular}} & 1.3793 \\
\hline \multicolumn{6}{|c|}{ Prot } \\
\hline 1 & $\begin{array}{l}\text { Johnson } \\
\text { SB }\end{array}$ & 0.08384 & & 4.1228 & N/A \\
\hline 2 & $\begin{array}{l}\text { Gen. } \\
\text { Pareto }\end{array}$ & 0.08441 & & 4.1348 & N/A \\
\hline
\end{tabular}




\section{CONCLUSION}

This paper attempts to estimate the pdf probability density function of various parameters of liver disease with cirrhosis or without cirrhosis. In this paper our objective is to apply best distribution over various parameters. Pdf is a function that describes the relative likelihood for random variable to take on a given value. Various distributions have been tested to get a good fit for most of the data. It is observed that there is different distribution for different parameters. Further work can be done to predict the main target by applying various methods.

\section{ACKNOWLEDGEMENT}

The authors would like to thank Department of Gastroenterology and Department of Herpetology, Nehru Hospital, PGIMER, Chandigarh for their support.

\section{REFERRENCES}

[1] W.B. Davenport, "A Study of Speech Probability Distribution" Ph.D. Thesis, Research Laboratory of Electronics, MIT, Cambridge, Mass., USA, August 1950

[2] Plebani J G, Tirado C F. Combined effects of alcohol and hepatitis $\mathrm{C}$ : a secondary analysis of alcohol use biomarkers and high-risk behaviors from two medication trials for alcohol dependence. Addict Behav 2010; 35(2): 123-8.

[3] Stroffolini T, Cotticelli G, Interaction of alcohol intake and cofactors on the risk of cirrhosis. Liver Int 2010; 30(6): 867-70.

[4] Robert S. O'Shea, Srinivasan Dasarathy, Arthur J. McCullough. Alcoholic liver disease. Hepatology 2010;51:307-328.

[5] S. Gazor, and W. Zhang, "Speech Probability Distribution" IEEE Signal Processing Letters, vol. 10, no. 7, July 2003, pp. 204-207

[6] Shifei Ding, Yanan Zhang, Jinrong Chen, Weikuan Jia "Research on using genetic algorithms to optimize Elman neural networks", Neural Comput \& Applic (2013) 23:293-297.

[7] Franzini, M. A. "Speech Recognition with Back Propagation", Proceedings of the IEEE/Ninth Annual Conference of the Engineering in Medicine and Biology Society, Boston, MA, 9, 1702-1703, 1987.

[8] Yan Sun, "Ultrasonographic classification of cirrhosis based on pyramid neural network", IEEE Electrical and Computer Engineerin, Canadian Conference 2005. 